\title{
CONVERGENCE OF A METHOD OF SOLVING LINEAR PROBLEMS1
}

W. KARUSH

1. Introduction. We are concerned with the solution of two problems associated with a linear operator $A$. First, the characteristic value problem

$$
A y=\lambda y
$$

for the determination of the characteristic values $\lambda$ and the characteristic vectors $y$; second, the linear equation problem

$$
(A-\lambda I) x=b,
$$

$$
b \neq 0,
$$

for the determination of $x$, given the number $\lambda$ and the vector $b$ ( $I$ is the identity operator). Lanczos $[3]^{2}$ has described an interesting iterative method for the solution of these problems which appears to be effective for numerical calculation. It is our purpose to consider the convergence and rate of convergence of the method, in the Hilbert space sense, for a bounded self-adjoint operator.

The procedure for obtaining the solution may be described as follows. Let $b \neq 0$ be a given initial vector, arbitrary for problem (1), equal to the right side of (2) for problem (2). Let

$$
\mathfrak{H C}_{i}=\left(b, A b, \cdots, A^{i-1} b\right) \text {, }
$$

i.e., the linear subspace spanned by the indicated vectors. Let $\mathfrak{T C}$ be the invariant subspace which is the closure of the linear subspace spanned by all non-negative powers $A^{i} b$; symbolically

$$
\mathfrak{H}=\left(b, A b, \cdots, A^{i} b, \cdots\right) .
$$

Let $\pi_{i}$ be the projection operator onto $\mathfrak{F}_{i}$. Then to solve (1) and (2) we replace the operator $A$ by the operator $\pi_{i} A$ on $\mathfrak{H C}_{i}$, solve the corresponding finite-dimensional problem, and allow $i$ to approach $\infty$. That is, (1) and (2) are approximated respectively by

$$
\pi_{i} A y=\lambda y
$$

on $\mathfrak{H}_{i}$

and

$$
\left(\pi_{i} A-\lambda I\right) x=b
$$

on $\mathfrak{H C}_{i}$.

Received by the editors February 13, 1952.

1 The preparation of this paper was sponsored (in part) by the Office of Naval Research.

2 Numbers in brackets refer to the list of references at the end of the paper. 
We shall establish the convergence of the solutions of (5) to a solution of (1) for isolated characteristic values; the convergence to the characteristic value is monotonic. This result applies only to solutions of (1) lying in $\mathfrak{F}$, of course. To prove the convergence of the solutions of (6) to the solution of (2) we make the assumption that $A$ is completely continuous. Under this latter hypothesis we are also able to show that the rate of convergence to the solution for each of the problems (1) and (2) is faster than any geometric progression.

One of the advantages of solving (1) and (2) by means of (5) and (6) is the simple algebraic form that the successive approximations take. For full details, as well as numerical examples, see the paper of Lanczos where self-adjointness is not necessarily assumed. Our description of the method in the preceding paragraph is somewhat different from Lanczos' formulation. The equivalence of the two procedures is shown in the final section of this paper.

Some additional remarks are in order. First, the present method should be contrasted with a procedure studied by the author in an earlier paper [2] in which only the characteristic value problem (1) was considered. In that paper a sequence of subspaces of fixed finite dimension was employed, each subspace depending upon the previous one, instead of the sequence of subspaces $\mathcal{H C}_{i}$ of increasing dimension described in (3). Further, the method of [2] is not immediately applicable to the linear equation problem (2). The second remark is that the present paper is related to the work of Rellich [4] in that we deal with successive approximations $\pi_{i} A$ to $A$. However it appears that the direct use here of his results would lead to no essential simplification. Finally, attention should be drawn to a paper of R. C. T. Smith [5] concerned with the calculation of characteristic values of infinite matrices. The matrix is approximated by its finite segments, that is, (1) is approximated by equation (5) with $\mathfrak{H}_{i}$ $=\left(e_{1}, e_{2}, \cdots, e_{i}\right), e_{j}$ being the sequence with 1 in the $j$ th place and zeros elsewhere. Explicit error estimates for the characteristic values are given.

2. Assumptions. In this section we shall specify the notation and assumptions which are to remain in force in the remainder of the paper.

We are given a bounded self-adjoint linear operator $A$ on a (not necessarily separable) real Hilbert space $R$ of infinite dimension. With a given vector $b$ we form the subspaces (3) and (4). Since our method of solution never leads us out of the invariant closed (separable) space $\mathfrak{H C}$, we henceforth understand by $A$ an operator on $\mathcal{H}$ 
(i.e., from $\mathfrak{F C}$ to $\mathfrak{F}$ ), unless otherwise stated. Thus we obtain only the characteristic solutions of (1) which belong to $\mathfrak{F}$; further solutions would be obtained by varying $b$. The hypotheses we shall make on $A$ will be hypotheses holding for $A$ as on $\mathfrak{K C}$; this is only a matter of convenience since it would be a simple matter to impose the conditions on $A$ as on $R$ and add a mild condition on $b$ to guarantee the satisfaction of the hypotheses of the theorems.

We understand always by $\pi_{i} A$ an operator on the subspace $\mathfrak{K C}_{i}$. As such $\pi_{i} A$ is self-adjoint; for, with $x$ and $y$ in $\mathfrak{H}_{i},\left(\pi_{i} A x, y\right)=(A x, y)$ $=(x, A y)=\left(x, \pi_{i} A y\right)$.

We shall make use of the Rayleigh quotient, which is defined by

$$
\mu(x)=\frac{(x, A x)}{(x, x)}, \quad x \neq 0 .
$$

For $x$ in $\mathfrak{F}_{i}$ we have $\left(x, \pi_{i} A x\right) /(x, x)=(x, A x) /(x, x)$, so that the Rayleigh quotient for the finite-dimensional operator $\pi_{i} A$ has the form (7) independent of $i$, provided $x$ is restricted to $\mathfrak{K C}_{i}$. As is well known the characteristic vectors of (5) are the minimax points for the function $\mu$ on $\mathfrak{F}_{i}[1$, pp. 27-29]. Certain other standard properties of $\mu$ will be taken as known.

Finally we assume as a matter of convenience that

$$
\text { dimension }\left(\mathfrak{F}_{i}\right)=i \text {. }
$$

That is, we assume that $\mathfrak{H C}$ is not finite-dimensional. If the contrary held, then, for some $k, \mathfrak{C}=\mathfrak{H C}_{k}$ and the method would terminate with an exact solution at the $k$ th step.

3. The characteristic value problem. It is easy to show that the vector $b$ has a non-null projection on every characteristic manifold of $A$, and that every such manifold has dimension one, i.e., every characteristic number is simple [2]. A similar statement holds for the operator $\pi_{i} A$, whose $i$ characteristic values we denote by

$$
\lambda_{1 i}>\lambda_{2 i}>\cdots>\lambda_{i i}
$$

and whose corresponding characteristic vectors we denote by

$$
y_{1 i}, y_{2 i}, \cdots, y_{i i}
$$

and which are taken to satisfy the conditions

$$
\left|y_{j i}\right|=1, \quad\left(b, y_{j i}\right)>0, \quad j=1,2, \cdots, i .
$$

For the next theorem we shall require the following assumption on the (real) spectrum $S$ of $A$.

(10) $\delta$ is the union of disjoint sets $\delta_{1}, \delta_{2}$ where $\delta_{1}$ consists of a 
finite number of isolated characteristic values

$$
\lambda_{1}>\lambda_{2}>\cdots>\lambda_{m}, \quad m \geqq 1,
$$

and $S_{2}$ is a closed set lying below $\lambda_{m}$.

This partitioning may be possible in many ways. The characteristic vectors of $A$ corresponding to the values in $\delta_{1}$ we denote by

$$
y_{1}, y_{2}, \cdots, y_{m}
$$

where

$$
\left|y_{j}\right|=1, \quad\left(b, y_{j}\right)>0, \quad j=1,2, \cdots, m .
$$

THEOREM 1. Let the spectrum of $A$ satisfy condition (10). For each $j, j=1,2, \cdots, m$, determine the infinite sequence $\left\{y_{j i}\right\}$ as above. Then $\left\{\lambda_{j i}\right\}$ is a monotonic increasing sequence, and

$$
\lim _{i \rightarrow \infty} y_{j i}=y_{j}, \quad \lim _{i \rightarrow \infty} \lambda_{j i}=\lambda_{j}, \quad j=1,2, \cdots, m .
$$

An entirely analogous result holds if the finite set $\delta_{1}$ lies below, instead of above, the remainder $S_{2}$ of the spectrum.

To make the proof we suppose first that the conclusion has been established for $j=1,2, \cdots, k-1,2 \leqq k \leqq m$; we show its validity for $j=k$. Observe that $\lambda_{k i}=\mu\left(y_{k i}\right)$ is a nondecreasing sequence, as follows from the minimax principle just referred to for characteristic values in finite-dimensional spaces. Further, the sequence is bounded by $\lambda_{1}$, since

$$
\lambda_{1}=\max \mu(x), \quad x \neq 0, x \in \mathcal{F} .
$$

Let $\bar{\lambda}$ be the limit of the sequence; we wish to show that $\bar{\lambda}=\lambda_{k}$.

Since $y_{k} \in \mathcal{H}$, it follows from the definition of $\mathfrak{F C}$ that there is a sequence of vectors $x_{i}, x_{i} \in \mathcal{F}_{i}$, such that $x_{i} \rightarrow y_{k}$ as $i \rightarrow \infty$. We may write

$$
x_{i}=\sum_{j=1}^{k-1} \beta_{j i} y_{j i}+r_{i}, \quad r_{i} \in \mathfrak{K}_{i},\left(r_{i}, y_{j i}\right)=0 .
$$

Take the inner product of each side of this equation with $y_{k i}$ and allow $i$ to tend to $\infty$. Then using the assumed limit relations and the orthogonality between characteristic vectors we find that $\beta_{j i} \rightarrow 0$. Thus $r_{i}$ tends to $y_{k}$, since $x_{i}$ does. Hence $\mu\left(r_{i}\right) \rightarrow \mu\left(y_{k}\right)=\lambda_{k}$. But $\mu\left(r_{i}\right) \leqq \mu\left(y_{k i}\right)$ since $y_{k i}$ maximizes $\mu(x)$ for $x \in \mathcal{F C}_{i}$ and $x$ orthogonal to $y_{j i}, j=1,2, \cdots, k-1$. Thus

$$
\lambda_{k} \leqq \bar{\lambda}
$$


To prove the reverse inequality we write

$$
y_{k i}=\sum_{j=1}^{k} \alpha_{j i} y_{j}+z_{i}, \quad z_{i} \in \mathcal{H C},\left(z_{i}, y_{j}\right)=0 .
$$

From (9) and (11)

$$
1=\sum_{j=1}^{k} \alpha_{j i}^{2}+\left|z_{i}\right|^{2}
$$

We also note the relation

$$
\lambda_{k}-\mu\left(z_{i}\right) \geqq \gamma>0,
$$

which follows from the fact that the least upper bound of the complement in $S$ of the finite set $\left(\lambda_{1}, \lambda_{2}, \cdots, \lambda_{k}\right)$ is the maximum of $\mu(x)$ for $x \in \mathcal{H},\left(x, y_{j}\right)=0, j=1,2, \cdots, k$. We take the inner product of each side of (12) with $\left(y_{n i}\right), n=1,2, \cdots, k-1$, and find that $\alpha_{n i} \rightarrow 0$ as $i \rightarrow \infty$. From (12) and the definition (7) of $\mu(x)$ we find that

$$
\mu\left(y_{k i}\right)=\sum_{j=1}^{k} \alpha_{j i \mu}^{2} \mu\left(y_{j}\right)+\mu\left(z_{i}\right)\left|z_{i}\right|^{2},
$$

that is,

$$
\lambda_{k i}=\sum_{j=1}^{h} \alpha_{j i}^{2} \lambda_{j}+\mu\left(z_{i}\right)\left|z_{i}\right|^{2} .
$$

Substituting for $\left|z_{i}\right|^{2}$ from (13) we obtain

$$
\begin{aligned}
\lambda_{k i} & =\sum_{j=1}^{k} \alpha_{j i}^{2}\left(\lambda_{i}-\mu\left(z_{i}\right)\right)+\mu\left(z_{i}\right) \\
& \leqq \mu\left(z_{i}\right)<\lambda_{k} .
\end{aligned}
$$

Thus

$$
\bar{\lambda} \leqq \lambda_{k}
$$

which completes the proof of $\bar{\lambda}=\lambda_{k}$.

To show the convergence of the vectors write (16) in the form

$$
\alpha_{k i}^{2}=1+\frac{1}{\lambda_{k}-\mu\left(z_{i}\right)}\left(\left(\lambda_{k i}-\lambda_{k}\right)-\sum_{j=1}^{k-1} \alpha_{j i}^{2}\left(\lambda_{j}-\mu\left(z_{i}\right)\right)\right) .
$$

Taking the limit and using (14) we obtain $\alpha_{z \rightarrow}^{2} \rightarrow 1$. From (13), $z_{i} \rightarrow 0$. The sequence $\alpha_{k i}$ cannot have the limit point -1 . If it did, a subsequence (denoted by the same subscript $i$ ) would converge to -1 . The corresponding subsequence $y_{k i}$ by (12) would converge to $-y_{k}$, 
hence $\left(b, y_{k i}\right) \rightarrow-\left(b, y_{k}\right)<0$ by (11), contrary to (9). Thus the original sequence $\alpha_{k i}$ tends to 1 and, by (12), $y_{k i} \rightarrow y_{k}$.

The proof will be complete if we justify the conclusion of the theorem for $j=1$. This justification is a duplication of the above proof with obvious simplifications. We omit the details.

4. Rate of convergence. In order to prove the convergence theorem for the solution of problem (2) we shall require some information on the rate of the convergence established in Theorem 1. This information is of independent interest as well. We now suppose that $A$ is completely continuous, although the results of this section could be formulated somewhat more generally. We shall show that the convergence to the solution of problem (1) is geometric with arbitrarily small ratio.

With $A$ completely continuous the spectrum of $A$ (omitting 0 ) consists of two sequences of characteristics values

$$
\begin{array}{ll}
\lambda_{1}>\lambda_{2}>\cdots, & \lambda_{j}>0, \\
\lambda_{1}^{\prime}<\lambda_{2}^{\prime}<\cdots, & \lambda_{j}^{\prime}<0,
\end{array}
$$

not both of which are finite or vacuous. Either infinite sequence has the limit 0 . In the sequel we shall speak as if both sequences are infinite. If one is finite or vacuous, then certain obvious notational changes should be made. Every characteristic value is simple and we denote corresponding characteristic vectors by

$$
\begin{aligned}
& y_{1}, y_{2}, \cdots, \\
& y_{1}^{\prime}, y_{2}^{\prime}, \cdots,
\end{aligned}
$$

normalized in length and direction by (11).

For problem (5) it will be convenient to denote the characteristic values (8) arranged in reverse order by

$$
\lambda_{1 i}^{\prime}<\lambda_{2 i}^{\prime}<\cdots<\lambda_{i i}^{\prime}
$$

with a similar notation for characteristic vectors. By Theorem 1 and the remark following it we have, for every positive integer $j$,

$$
y_{j i} \rightarrow y_{j}, \lambda_{j i} \rightarrow \lambda_{j}, \quad y_{j i}^{\prime} \rightarrow y_{j}^{\prime}, \lambda_{j i}^{\prime} \rightarrow \lambda_{j}^{\prime} .
$$

Theorem 2. Suppose that $A$ is completely continuous. Let $\delta$ be an arbitrary number with $0<\delta<1$. Then for each characteristic number $\lambda_{k}\left(\lambda_{k}^{\prime}\right)$ there is a constant $K\left(K^{\prime}\right)$ independent of $i$ such that

$$
\left|\lambda_{k}-\lambda_{k i}\right| \leqq K \delta^{i} \quad\left(\left|\lambda_{k}^{\prime}-\lambda_{k i}^{\prime}\right|<K^{\prime} \delta^{i}\right) .
$$


We give the proof for a positive value $\lambda_{k}$; for a negative value we need only notice that the proof is entirely analogous or alternatively we may transform to the operator $-A$.

We choose positive integers $m, m^{\prime}$ so that

$$
\max \left(\frac{\lambda_{m+1}}{\lambda_{k}}, \frac{\left|\lambda_{m^{\prime}+1}^{\prime}\right|}{\lambda_{k}}\right)<\delta .
$$

We write

$$
b=\sum_{j=1}^{m} \alpha_{j} y_{j}+\sum_{j=1}^{m^{\prime}} \alpha_{j}^{\prime} y_{j}^{\prime}+z
$$

with $z$ orthogonal to the subspace

$$
\mathcal{L}=\left(y_{1}, \cdots, y_{m}, y_{1}^{\prime}, \cdots, y_{m}^{\prime}\right) \text {. }
$$

By (11) we have

$$
\alpha_{j}>0, \quad \alpha_{j}^{\prime}>0
$$

Also

$$
b=\sum_{j=1}^{k-1} \alpha_{j i} y_{j i}+z_{i}, \quad z_{i} \in \mathcal{H}_{i},\left(z_{i}, y_{j i}\right)=0 .
$$

Let $q(\lambda)$ be an arbitrary polynomial of degree $i-1$. From (18)

$$
x_{i} \equiv q(A) b=\sum_{j=1}^{m} \alpha_{j} q\left(\lambda_{j}\right) y_{j}+\sum_{j=1}^{m^{\prime}} \alpha_{j}^{\prime} q\left(\lambda_{j}^{\prime}\right) y_{j}^{\prime}+q(A) z, \quad x_{i} \in \mathcal{F}_{i} .
$$

Now $q(A) b=q\left(\pi_{i} A\right) b$. Hence, from (20), $x_{i}=\sum_{j=1}^{k-1} \alpha_{j i} q\left(\pi_{i} A\right) y_{j i}$ $+q\left(\pi_{i} A\right) z_{i}$, so that

$$
x_{i}=\sum_{j=1}^{k-1} \alpha_{j i} q\left(\lambda_{j i}\right) y_{j i}+\bar{z}_{i}, \quad \bar{z}_{i} \in \mathcal{H C}_{i},\left(\bar{z}_{i}, y_{j i}\right)=0 .
$$

We choose

$$
\begin{aligned}
q(\lambda)=\lambda^{*} \prod_{j=1, j \neq k}^{m}\left(\lambda-\lambda_{j}\right) \prod_{j=1}^{m^{\prime}}\left(\lambda-\lambda_{j}^{\prime}\right) \prod_{j=1}^{k-1}\left(\lambda-\lambda_{j i}\right), \\
s=i-m-m^{\prime}-k+1 .
\end{aligned}
$$

For $i$ sufficiently large $q$ is well-defined and $q\left(\lambda_{k}\right) \neq 0$. By (22), we have $x_{i}=\bar{z}_{i}$. Hence

$$
\mu\left(x_{i}\right) \leqq \lambda_{k i},
$$


since $\lambda_{k i}=\max \mu(x)$ for $x \in \mathcal{F C}_{i}, x \neq 0$ orthogonal to $y_{j i}, j=1,2, \cdots$, $k-1$. By (21)

$$
x_{i}=\alpha_{k} q\left(\lambda_{k}\right) y_{k}+r_{i}, \quad r_{i}=q(A) z .
$$

Thus

$$
\begin{aligned}
\mu\left(x_{i}\right) & =\frac{\alpha_{k}^{2} q^{2}\left(\lambda_{k}\right) \lambda_{k}+\mu\left(r_{i}\right)\left|r_{i}\right|^{2}}{\alpha_{k}^{2} q^{2}\left(\lambda_{k}\right)+\left|r_{i}\right|^{2}}, \\
\lambda_{k}-\mu\left(x_{i}\right) & =\frac{\left(\lambda_{k}-\mu\left(r_{i}\right)\right)\left|r_{i}\right|^{2}}{\alpha_{k}^{2} q^{2}\left(\lambda_{k}\right)+\left|r_{i}\right|^{2}} \leqq K_{1} \frac{\left|r_{i}\right|^{2}}{q^{2}\left(\lambda_{k}\right)} .
\end{aligned}
$$

To estimate the last ratio we use the fact that $z$ is orthogonal to $\mathcal{L}$. From the spectral resolution of $A$,

$$
r_{i}=\int_{S} q(\lambda) d E(\lambda) z=\int_{\lambda_{m^{\prime}+1}^{\prime}}^{\lambda_{m+1}} q(\lambda) d E(\lambda) z .
$$

Hence

$$
\left|r_{i}\right|^{2}=\int_{\lambda_{m^{\prime}+1}^{\prime}}^{\lambda_{m+1}} q^{2}(\lambda) d|E(\lambda) z|^{2} \leqq q^{2}(\bar{\lambda})|z|^{2}, \quad \lambda_{m^{\prime}+1}^{\prime} \leqq \bar{\lambda} \leqq \lambda_{m+1} .
$$

Utilizing the definition of $q(\lambda)$ and (17) we obtain from the preceding inequality, for $i$ sufficiently large,

$$
\frac{\left|r_{i}\right|^{2}}{q^{2}\left(\lambda_{k}\right)} \leqq|z|^{2} \frac{q^{2}(\bar{\lambda})}{q^{2}\left(\lambda_{k}\right)} \leqq K_{2}\left(\frac{\bar{\lambda}^{2}}{\lambda_{k}^{2}}\right) \leqq K_{3} \delta^{i}
$$

Combining this with (24) yields

$$
\lambda_{k}-\mu\left(x_{i}\right) \leqq K \delta^{i} .
$$

Finally, from (23), $\lambda_{k}-\lambda_{k i} \leqq \lambda_{k}-\mu\left(x_{i}\right)$. The conclusion of the theorem now follows from the fact that $\lambda_{k i}$ approaches $\lambda_{k}$ monotonically from below.

For the convergence of the characteristic vectors a similar estimate holds. We state the result without proof, for we shall not require it in the sequel. The proof is made by re-doing the proof of Theorem 1 with the added result in Theorem 2.

THEOREM 3. Under the hypotheses of Theorem 2 there is for each characteristic vector $y_{k}\left(y_{k}^{\prime}\right)$ a constant $K\left(K^{\prime}\right)$ independent of $i$ such that

$$
\left|y_{k}-y_{k i}\right| \leqq K \delta^{i} \quad\left(\left|y_{k}^{\prime}-y_{k i}^{\prime}\right| \leqq K^{\prime} \delta^{i}\right)
$$


5. The linear equation problem. We turn now to the solution of problem (2), where the given number $\lambda$ is assumed to be in the resolvent set of $A$ and the given vector $b$ is, of course, to be used in the construction of the spaces (3) and (4). Then a unique solution

$$
x_{0}=(A-\lambda I)^{-1} b
$$

in TC exists. As an aside we remark that if $A$ is regarded as an operator on the original space $R$, then the assumption that $\lambda$ is in the resolvent set of $A$ as on $R$ implies that $\lambda$ is in the resolvent set of $A$ as on 3 . But the reverse implication does not hold; for example, $b$ may be orthogonal to a particular characteristic manifold of $A$ as on $R$. Thus, imposing our condition on $A$ as on $\mathcal{H C}$ represents a weaker assumption.

As in the preceding section we suppose that $A$ is completely continuous, for we shall require the results of Theorem 2 . Since $\lambda$ is in the resolvent set of $A$, we have

$$
\lambda \neq 0
$$

and by Theorem 1 it follows that for $i$ sufficiently large the number $\lambda$ is not characteristic for $\pi_{i} A$. Thus equation (5) has a unique solution $x_{i}$, i.e.,

$$
\pi_{i}(A-\lambda I) x_{i}=b, \quad x_{i} \in \mathcal{F}_{i} .
$$

We shall establish the convergence of $x_{i}$ to the solution (25) of equation (2), and estimate the rate of convergence.

Let

$$
\phi_{i}(\lambda)=\lambda^{i}+\gamma_{1} \lambda^{i-1}+\cdots+\gamma_{i}=\prod_{j=1}^{i}\left(\lambda-\lambda_{j i}\right)
$$

be the characteristic polynomial of $\pi_{i} A$ (for simplicity we do not indicate the dependence of the coefficients $\gamma$ on the index $i$ ). We have $0=\phi_{i}\left(\pi_{i} A\right) b=\pi_{i} A^{i} b+\gamma_{1} A^{i-1} b+\cdots+\gamma_{i} b=\phi_{i}(A) b-\left(A^{i} b-\pi_{i} A^{i} b\right)$. Thus $\phi_{i}(A) b=A^{i} b-\pi_{i} A^{i} b$, so that if we let

$$
\xi_{i}=\phi_{i}(A) b
$$

then $\xi_{i} \in \mathcal{F C}_{i+1}$ and $\pi_{i} \xi_{i}=0$. From (27)

$$
A^{i} b=\xi_{i}-\gamma_{1} A^{i-1} b-\cdots-\gamma_{i} b .
$$

If we represent the solution $x_{i}$ in the form

$$
x_{i}=\beta_{0} b+\beta_{1} A b+\cdots+\beta_{i-1} A^{i-1} b,
$$

then Lanczos [3] has given the solution 


$$
\begin{aligned}
\beta_{i-1} & =-\frac{1}{\phi_{i}(\lambda)}, \quad \beta_{i-2}=-\frac{\lambda+\gamma_{1}}{\phi_{i}(\lambda)}, \cdots, \\
\beta_{1} & =-\frac{\lambda^{i-2}+\gamma_{1} \lambda^{i-3}+\cdots+\gamma_{i-2}}{\phi_{i}(\lambda)} \\
\beta_{0} & =-\frac{\lambda^{i-1}+\gamma_{1} \lambda^{i-2}+\cdots+\gamma_{i-1}}{\phi_{i}(\lambda)} .
\end{aligned}
$$

To verify this, we substitute (29) into $b-(A-\lambda I) x_{i}$, using (30) for the values of $\beta$ and replacing $A^{i} b$ by the expression on the right of (28). The result is

$$
b-(A-\lambda I) x_{i}=\xi_{i} / \phi_{i}(\lambda) .
$$

Since $\pi_{i} \xi_{i}=0$, this equation tells us that $x_{i}$ is the solution of (26); further

$$
r_{i} \equiv b-(A-\lambda I) x_{i}=\frac{\phi_{i}(A) b}{\phi_{i}(\lambda)} .
$$

We now state the convergence theorem.

Theorem 4. Suppose that $A$ is completely continuous. Let $\lambda \neq 0$ be noncharacteristic for $A$, and let $x_{0}$ be the unique solution in $\mathrm{HC}$ of equation (2). Let $\delta$ be an arbitrary number with $0<\delta<1$. Then for sufficiently large $i$ equation (6) has a unique solution $x_{i}$ in $\mathcal{F C}_{i}$, and there exists a constant $K$ independent of $i$ such that

$$
\left|x_{i}-x_{0}\right| \leqq K \delta^{i}
$$

In particular, $\lim _{i \rightarrow \infty} x_{i}=x_{0}$.

We have already remarked that there exists a unique $x_{i}$ for sufficiently large $i$. To establish (32) we first choose $\alpha>0$ so that

$$
\alpha<|\lambda| \text { and } \frac{2 \alpha}{|\lambda|-\alpha}<\delta \text {. }
$$

Using the notation of the preceding section choose the indices $m$ and $m^{\prime}$ so that $\lambda_{m+1}$ and $\lambda_{m^{\prime}+1}^{\prime}$ lie in the closed interval $[-\alpha, \alpha]$. We express $b$ in the form (18) and consider the remainder $r_{i}$ of (31). We have

$$
r_{i}=\frac{\phi_{i}(A) b}{\phi_{i}(\lambda)}=\sum_{j=1}^{m} \frac{\phi_{i}\left(\lambda_{j}\right)}{\phi_{i}(\lambda)} \alpha_{j} y_{j}+\sum_{j=1}^{m^{\prime}} \frac{\phi_{i}\left(\lambda_{j}^{\prime}\right)}{\phi_{i}(\lambda)} \alpha_{j}^{\prime} y_{j}^{\prime}+\frac{\phi_{i}(A) z}{\phi_{i}(\lambda)}
$$


Let

$$
\sigma=2^{-1} \min |\lambda-\nu|>0, \quad M=2 \max |\nu|>0 \quad \text { for } \nu \in S .
$$

Choose $\delta_{1}, 0<\delta_{1}<1$, so that

$$
\delta_{1} M / \sigma<\delta .
$$

We now consider the coefficient $\phi_{i}\left(\lambda_{j}\right) / \phi_{i}(\lambda)$ in (34). Apply Theorem 2 to $\lambda_{j}$ for the value $\delta_{1}$ to obtain an appropriate constant $K_{1}$. Then for sufficiently large $i$ we obtain, by writing $\phi_{i}$ in factored form,

$$
\left|\frac{\phi_{i}\left(\lambda_{j}\right)}{\phi_{i}(\lambda)}\right| \leqq\left|\lambda_{j}-\lambda_{j i}\right| \frac{M^{i-1}}{\sigma^{i}} \leqq \frac{K_{1}}{M}\left(\frac{\delta_{1} M}{\sigma}\right)^{i} \leqq K \delta^{i}
$$

Similarly, we obtain the same inequality for $\lambda_{j}^{\prime}$.

Finally we treat the last term on the right of (34). We have

$$
\left|\frac{\phi_{i}(A) z}{\phi_{i}(\lambda)}\right|=\left|\frac{1}{\phi_{i}(\lambda)} \int_{-\alpha}^{\alpha} \phi_{i}(\nu) d E(\nu) z\right| \leqq\left|\frac{\phi_{i}(\bar{\lambda})}{\phi_{i}(\lambda)}\right| \cdot|z|, \quad \bar{\lambda} \text { in }[-\alpha, \alpha] .
$$

For $i$ sufficiently large all roots $\lambda_{k i}, k=m+1, m+2, \cdots$, and $\lambda_{k t}^{\prime}, k=m^{\prime}+1, m^{\prime}+2, \cdots$, lie in $[-\alpha, \alpha]$. Hence, writing $\phi_{i}(\lambda)$ in factored form, we find

$$
\left|\frac{\phi_{i}(\bar{\lambda})}{\phi_{i}(\lambda)}\right| \leqq\left(\frac{M}{\sigma}\right)^{m+m^{\prime}}\left(\frac{2 \alpha}{|\lambda|-\alpha}\right)^{i-\left(m+m^{\prime}\right)} \leqq K \delta^{i}
$$

by (33). From the preceding inequalities and equation (34),

$$
\left|r_{i}\right| \leqq K \delta^{i} \text {. }
$$

From (31) $x_{i}=B\left(b-r_{i}\right)=x_{0}-B r_{i}$ where $B=(A-\lambda I)^{-1}$ is a bounded operator. Thus

$$
\left|x_{i}-x_{0}\right| \leqq\left|B r_{i}\right| \leqq K \delta^{i}
$$

for an appropriate constant $K$. This completes the proof.

6. Equivalence with procedure of Lanczos. In order to show that our method is identical with that of Lanczos we shall reproduce some of his formulas here (see $[3, \S \S \mathrm{VII}$ and $\mathrm{X}]$ ). His notation has been modified slightly to conform with ours; in particular, his $\mu=1 / \lambda$ becomes our $\lambda$. Beginning with the initial vector $b=b_{0}$, constants $\alpha_{j}, \beta_{j}$, polynomials $p_{j}(\lambda)$, and vectors $b_{j}$ are defined recursively by

$$
\begin{aligned}
\alpha_{j} & =\mu\left(b_{j}\right), \quad \beta_{j}=\left|b_{j+1}\right|^{2} /\left|b_{j}\right|^{2} \\
p_{0}(\lambda) & \equiv 1, p_{1}(\lambda)=\lambda-\alpha_{0}, p_{j+1}(\lambda)=\left(\lambda-\alpha_{j}\right) p_{j}(\lambda)-\beta_{j-1} p_{j-1}(\lambda), \\
b_{j} & =p_{j}(A) b_{0}, \quad b_{j} \in \mathcal{H}_{j+1} .
\end{aligned}
$$


These quantities are defined in the order $b_{j}, \alpha_{j}, \beta_{j-1}, p_{j+1}(\lambda), b_{j+1}$. Let us show first that the polynomial $p_{i}(\lambda)$ is the characteristic polynomial of the operator $\pi_{i} A$ on $\mathfrak{F C}_{i}$. Lanczos shows (pp. 266-267) that $b_{i}$ is that vector of the form $A^{i} b-z, z \in \mathcal{F}_{i}$, which has minimum length. Clearly this vector must be given by

$$
b_{i}=A^{i} b_{0}-\pi_{i} A^{i} b_{0} .
$$

Now let $\phi_{i}(\lambda)$ be the characteristic function of $\pi_{i} A$. Then $0=\phi_{i}\left(\pi_{i} A\right) b_{0}$ $=-A^{i} b_{0}+\pi_{i} A^{i} b_{0}+\phi_{i}(A) b_{0}=-b_{i}+\phi_{i}(A) b_{0}$. Hence, by the last equation (35), $\phi_{i}(A) b_{0}=p_{i}(A) b_{0}$, so that $\phi_{i}(\lambda) \equiv p_{i}(\lambda)$, since $\mathcal{H}_{i}$ has dimension $i$. Thus Lanczos' approximation to the characteristic numbers of $A$, namely the roots of $p_{i}(\lambda)$, is identical with our approximation.

His approximation to the $j$ th characteristic vector (see his equation $(107))$ is

$$
y=\sum_{k=0}^{i-1} \frac{p_{k}\left(\lambda_{j}\right)}{\left|b_{k}\right|^{2}} b_{k},
$$

where $\lambda_{j}$ is the $j$ th root of $p_{i}(\lambda)=0$. We need to verify that this vector is the $j$ th characteristic vector of $\pi_{i} A$, i.e., that

$$
\pi_{i} A y=\lambda_{i} y_{j} .
$$

To this end we rewrite the recursion formula for the polynomials $p(\lambda)$ in the form

$$
\lambda p_{k}(\lambda)=p_{k+1}(\lambda)+\alpha_{k} p_{k}(\lambda)+\beta_{k-1} p_{k-1}(\lambda), \quad p_{-1}(\lambda) \equiv 0 .
$$

Replacing $\lambda$ by $A$ and operating on $b_{0}$ leads to

$$
\begin{aligned}
A b_{0} & =b_{1}+\alpha_{0} b_{0}, \\
A b_{k} & =b_{k+1}+\alpha_{k} b_{k}+\beta_{k-1} b_{k-1}, \quad k=1,2, \cdots, i-2, \\
\pi_{i} A b_{i-1} & =\alpha_{i-1} b_{i-1}+\beta_{i-2} b_{i-2} .
\end{aligned}
$$

Substituting the value of $y$ in (36) into the left side of (37) and reducing the resulting expression to a linear combination of the $b_{k}$ 's by means of (38) leads to the right side of (37). The required verification is thereby accomplished.

Finally, we need to show that his approximation to the solution of equation (2) is the solution of equation (6). With appropriate notational changes his approximation is (see his equation (102))

$$
x=-\frac{1}{p_{i}(\lambda)} \sum_{k=0}^{i-1} p_{i-k-1}(\lambda) b_{k}
$$


where

$$
\begin{aligned}
& p_{0}(\lambda) \equiv 1, \quad p_{1}(\lambda)=\lambda-\alpha_{i-1}, \\
& p_{k}(\lambda)=\left(\lambda-\alpha_{i-k}\right) p_{k-1}(\lambda)-\beta_{i-k} \bar{p}_{k-2}(\lambda) .
\end{aligned}
$$

Again, direct substitution of the value of $x$ in (39) into the left side of (6) and use of (38) and the recursion relation for the polynomials $\not(\lambda)$ leads to the right side of (6), establishing the desired result.

\section{REFERENCES}

1. R. Courant and D. Hilbert, Methoden der Mathematischen Physik, vol.1, 2d ed., Berlin, 1931.

2. W. Karush, Determination of the extreme values of the spectrum of a bounded selfadjoint operator, Proceedings of the American Mathematical Society vol. 2 (1951) pp. 980-989.

3. C. Lanczos, An iteration method for the solution of the eigenvalue problem of linear differential and integral operators, J. Research NBS vol. 45 (1950) pp. 255-282.

4. F. Rellich, Störungstheorie der Spektralzerlegung. II, Math. Ann. vol. 113 (1936) pp. $677-685$.

5. R. C. T. Smith, The approximate solution of equations in infinitely many unknowns, Quart. J. Math. vol. 18 (1947) pp. 25-52.

National Bureau of Standards, Los Angeles and

University of Chicago 